\title{
Open system of interacting fermions: Statistical properties of cross sections and fluctuations
}

\author{
G.L. Celardo ${ }^{1}$ F.M. Izrailev, ${ }^{1}$ V.G. Zelevinsky, ${ }^{2}$ and G.P. Berman ${ }^{3}$ \\ ${ }^{1}$ Instituto de Física, Universidad Autónoma de Puebla, \\ Apartado Postal J-48, Puebla, Pue., 72570, México \\ 2 NSCL and Department of Physics and Astronomy, \\ Michigan State University, East Lansing, Michigan 48824-1321, USA. \\ 3 Theoretical Division and CNLS, Los Alamos National Laboratory, Los Alamos, New Mexico 87545, USA.
}

(Dated: October 5, 2018)

\begin{abstract}
Statistical properties of cross sections are studied for an open system of interacting fermions. The description is based on the effective non-Hermitian Hamiltonian that accounts for the existence of open decay channels preserving the unitarity of the scattering matrix. The intrinsic interaction is modelled by the two-body random ensemble of variable strength. In particular, the crossover region from isolated to overlapping resonances accompanied by the effect of the width redistribution creating super-radiant and trapped states is studied in detail. The important observables, such as average cross section, its fluctuations, autocorrelation functions of the cross section and scattering matrix, are very sensitive to the coupling of the intrinsic states to the continuum around the crossover. A detailed comparison is made of our results with standard predictions of statistical theory of cross sections, such as the Hauser-Feshbach formula for the average cross section and Ericson theory of fluctuations and correlations of cross sections. Strong deviations are found in the crossover region, along with the dependence on intrinsic interactions and degree of chaos inside the system.
\end{abstract}

PACS numbers: 05.50.+q, 75.10.Hk, 75.10.Pq

\section{INTRODUCTION}

Information on properties of quantum mesoscopic systems comes mostly from various reactions where the system plays a role of a target. At high density of intrinsic states, the dynamics of realistic systems of interacting constituents becomes chaotic. Onset of chaos immensely complicates the details of the scattering process as reflected in the paradigm of compound nucleus. At low energies, the long-lived resonance states are exceedingly complex superpositions in the basis of independent particles. As energy increases, the scattering pattern evolves from the set of narrow isolated resonances to overlapping resonances and strongly fluctuating cross sections. Since the individual properties of resonances cannot be predicted, only statistical description is practical and sensible. The average cross sections are usually described according to Hauser-Feshbach [1], while the fluctuations and correlations of cross sections are treated in terms of Ericson theory [2, 3, 4] .

Standard theory of statistical reactions does not answer the question of interplay between reactions and internal structure determined by the character of interactions between the constituents. Here more detailed considerations are required based on the generalization of the shell model of nuclear reactions [5]. Such an extension introduces statistical assumptions concerning intrinsic dynamics and its coupling to the continuum [6, 7] with the extreme limiting case of intrinsic chaos modelled by the Gaussian Orthogonal Ensemble (GOE) [8]. An important element is still missing here, namely the transitional regime from separated to overlapping resonances. The consistent description based on the continuum shell model [5], as well as more phenomenological approaches 9, 10, 11], indicate the presence of a sharp restructuring of the system when the widths of resonances become comparable to their energy spacings. This phenomenon carries features of a quantum phase transition with the strength of continuum coupling playing the role of a control parameter.

As was clearly observed in the shell model framework 12], the distribution of resonance widths rapidly changes in the transitional region in such a way that a number of very broad resonances equal to a number of open decay channels absorb the lion's share of the total width of all overlapped resonances, while the remaining states become very narrow. The corresponding theory was suggested in [13, 14, 15], where the mechanism of this restructuring was understood to be associated with the nature of the effective non-Hermitian Hamiltonian [5] that describes the intrinsic dynamics after eliminating the channel variables. The factorized structure of this Hamiltonian, in turn, is dictated by the unitarity of the scattering matrix [16]. One can compare this phenomenon to the classical factorized model [17] of a giant resonance, where the collective strength of many particle-hole states is shifted in energy and concentrated at a specific combination of excited states. In spite of formal analogy, physics under study here is different. The concentration of widths on a few broad states can be described as collectivization along the imaginary axis in the complex energy plane. The driving force of this restructuring is the presence of open decay channels and interaction of intrinsic states through the continuum. The intrinsic Hermitian interaction is present as well and should be fully accounted for; one of the goals of our study is to under- 
stand the dependence of the continuum picture on the strength and character of interactions inside the closed system. The interplay of two collectivities is an interesting subject [18, 19] practically important in relation to the so-called pygmy-resonances in loosely bound systems [20].

The segregation of short-lived broad resonances from long-lived trapped states was shown to be similar to the superradiance [21] in quantum optics induced by the coupling of atomic radiators through the common radiation field, an analog of coherent coupling of many overlapped intrinsic states through continuum. Later a general character of the phenomenon was demonstrated for systems with GOE intrinsic dynamics and many open channels [22, 23]. Modern versions of the shell model in continuum [24, 25] are based on the effective Hamiltonian and naturally reveal the superradiance phenomenon as an important element. The transition to this regime should be taken into account in all cases when a physical system is strongly coupled to the continuum, see for example [26] and references therein.

The segregation of scales is spectacularly seen in level and width statistics [14, 22, 27, 28]. Even for GOE intrinsic dynamics, the probability $P(s \rightarrow 0)$ of very small spacings between the centroids of resonances does not vanish because of energy uncertainty of unstable states. The width distribution reveals the separation of the "cloud" of superradiant states far from the real energy axis, while the trapped states are clearly accumulated near the real axis. Some features of the picture are however sensitive to the character and strength of intrinsic interactions as we have demonstrated recently [29]. The analysis included the two-body random ensemble of variable interaction strength in a Fermi system of shell-model type and the GOE as an extreme limit of many-body random interaction.

In the present work, using the same framework as in [29], we study the interplay between the intrinsic dynamics and statistical properties of cross sections comparing the results with those of conventional approaches, namely Hauser-Feshbach average cross sections and Ericson fluctuations and correlations. In particular, we show that the assumption that fluctuations of the resonance widths are negligible for a large number of channels is not correct. We also show that the elastic enhancement factor strongly depends on the degree of chaoticity inside the system, thus leading to deviations from the HauserFeshbach formula.

\section{THE MODEL}

\section{A. Hamiltonian}

We consider a system of $n$ interacting fermions on $m$ mean-field orbitals. A large number, $N=m ! /[n !(m-$ $n)$ !], of intrinsic many-body states $|i\rangle$ comprise our Hilbert space. In our simulations we take $n=6, m=12$ that provides a sufficiently large dimension $N=924$. The states are unstable being coupled to $M$ open decay channels. The dynamics of the whole system is governed by an effective non-Hermitian Hamiltonian [5, 14, 26] given by a sum of two $N \times N$ matrices,

$$
\mathcal{H}=H-\frac{i}{2} W ; \quad W_{i j}=\sum_{c=1}^{M} A_{i}^{c} A_{j}^{c} .
$$

Here and below the intrinsic many-body states are labelled as $i, j, \ldots$ and decay channels as $a, b, c \ldots$. In Eq. (1), $H$ describes Hermitian internal dynamics that in reality also can be influenced by the presence of the continuum [24, 25, 30], while $W$ is a sum of terms factorized in amplitudes $A_{i}^{c}$ coupling intrinsic states $|i\rangle$ to the channels $c$. Under time-reversal invariance, these amplitudes can be taken as real quantities so that both $H$ and $W$ are real symmetric matrices.

We model $H$ by the two-body random ensemble (TBRE) assuming the intrinsic Hamiltonian $H$ in the form $H=H_{0}+V$, where $H_{0}$ describes the mean-field single-particle levels $|\nu\rangle$, and $V$ is a random two-body interaction between the particles [31]. The single-particle energies, $\epsilon_{\nu}$, are assumed to have a Poissonian distribution of spacings, with the mean level density $1 / d_{0}$. The interaction $V$ is characterized by the variance of the twobody random matrix elements, $\left\langle V_{\nu_{1}, \nu_{2} ; \nu_{3}, \nu_{4}}^{2}\right\rangle=v_{0}^{2}$. With no interaction, $v_{0}=0$, the many-body states have also the Poissonian spacing distribution $P(s)$. In the opposite extreme limit, $d_{0}=0$, corresponding to infinitely strong interaction, $\lambda \equiv v_{0} / d_{0} \rightarrow \infty$, the function $P(s)$ is close to the Wigner-Dyson (WD) distribution typical for a chaotic system [8]. Following Ref. [31], the critical interaction for the onset of strong chaos can be estimated as

$$
\lambda_{\mathrm{cr}}=\frac{v_{\mathrm{cr}}}{d_{0}} \approx \frac{2(m-n)}{N_{s}},
$$

where $N_{s}=n(m-n)+n(n-1)(m-n)(m-n-1) / 4$ is the number of directly coupled many-body states in any row of the matrix $H_{i j}$. Thus, we have $\lambda_{\text {cr }} \approx 1 / 20$, and often we perform the simulations with the value $\lambda=1 / 30$ slightly lower than $\lambda_{\mathrm{cr}}$. In parallel, we also consider the intrinsic Hamiltonian $H$ belonging to the GOE that corresponds to a many-body interaction, when the matrix elements are Gaussian random variables, $\left\langle H_{i j}^{2}\right\rangle=1 / N$ for $i \neq j$ and $\left\langle H_{i j}^{2}\right\rangle=2 / N$ for $i=j$.

The real amplitudes $A_{i}^{c}$ are assumed to be random independent Gaussian variables with zero mean and variance

$$
\left\langle A_{i}^{c} A_{j}^{c^{\prime}}\right\rangle=\delta_{i j} \delta^{c c^{\prime}} \frac{\gamma^{c}}{N} .
$$

The parameters $\gamma^{c}$ with dimension of energy characterize the total coupling of all states to the channel $c$. The normalization used in Eq. (3) is convenient if the energy interval $N D$ covered by decaying states is finite. Here $D$ 
is the distance between the many-body states in the middle of the spectrum, $D=1 / \rho(0)$, where $\rho(E)$ is the level density, and $E=0$ corresponds to the center of the spectrum. We neglect a possible explicit energy dependence of the amplitudes that is important near thresholds and is taken into account in realistic shell model calculations [25, 30]. The ratio $\gamma^{c} / N D$ characterizes the degree of overlap of the resonances in the channel $c$. We define the corresponding control parameter as

$$
\kappa^{c}=\frac{\pi \gamma^{c}}{2 N D}
$$

The transitional region corresponds to $\kappa^{c} \approx 1$. Varying the intrinsic interaction and, therefore, the level density $\rho$, we renormalize correspondingly the absolute magnitude of the widths, $\gamma$, in order to keep the coupling to continuum given by Eq. (4) fixed.

\section{B. Scattering matrix}

The effective Hamiltonian allows one to study the cross sections for possible reactions $b \rightarrow a$,

$$
\sigma^{b a}(E)=\left|\mathcal{T}^{b a}(E)\right|^{2}
$$

(our cross sections are dimensionless since we omit the common factor $\pi / k^{2}$ ). In what follows we study both the elastic, $b=a$, and non-elastic, $b \neq a$, cross sections. Ignoring the smooth potential phases irrelevant for our purposes we express the scattering amplitude of the reaction, $\mathcal{T}^{b a}$, in terms of the amplitudes $A_{i}^{c}$,

$$
\mathcal{T}^{b a}(E)=\sum_{i, j}^{N} A_{i}^{b}\left(\frac{1}{E-\mathcal{H}}\right)_{i j} A_{j}^{a} .
$$

Here the denominator contains the total effective Hamiltonian (1) including in this way the continuum coupling $W$ to all orders.

We can also write $\mathcal{T}^{b a}(E)$ in a different way, diagonalizing the effective non-Hermitian Hamiltonian $\mathcal{H}$. Its eigenfunctions $|r\rangle$ and $\langle\tilde{r}|$ form a bi-orthogonal complete set,

$$
\mathcal{H}|r\rangle=\mathcal{E}_{r}|r\rangle, \quad\langle\tilde{r}| \mathcal{H}=\langle\tilde{r}| \mathcal{E}_{r}^{*},
$$

and its eigenvalues are complex energies,

$$
\mathcal{E}_{r}=E_{r}-\frac{i}{2} \Gamma_{r}
$$

corresponding to the resonances with centroids $E_{r}$ and widths $\Gamma_{r}$. The decay amplitudes $A_{i}^{b}$ are transformed according to

$$
\mathcal{A}_{r}^{b}=\sum_{i} A_{i}^{b}\langle i \mid r\rangle, \quad \tilde{\mathcal{A}}_{r}^{a}=\sum_{j}\langle\tilde{r} \mid j\rangle A_{j}^{a},
$$

and the transition amplitudes are given by

$$
\mathcal{T}^{b a}(E)=\sum_{r}^{N} \mathcal{A}_{r}^{b} \frac{1}{E-\mathcal{E}_{r}} \tilde{\mathcal{A}}_{r}^{a},
$$

The bi-orthogonality of the transformation ensures that the statistical properties (3) of the ensemble of the amplitudes are preserved,

$$
\left\langle\tilde{\mathcal{A}}_{r}^{a} \mathcal{A}_{r^{\prime}}^{b}\right\rangle=\delta^{a b} \delta_{r r^{\prime}} \frac{\gamma^{a}}{N} .
$$

Introducing the matrix in channel space analogous to what is routinely used in the resonance data analysis,

$$
K=\frac{1}{2} \mathbf{A} \frac{1}{E-H} \mathbf{A}^{T},
$$

where the denominator includes only intrinsic dynamics and $\mathbf{A}$ is the $N \times M$ matrix of the transition amplitudes $A_{j}^{c}$, one can relate it to the transition amplitude $\mathcal{T}$ and the scattering matrix,

$$
S^{b a}=\delta^{b a}-i \mathcal{T}^{b a},
$$

in the explicitly unitary form

$$
S=\frac{1-i K}{1+i K}
$$

The poles of the $K$-matrix are real eigenvalues $E_{\alpha}$ of the intrinsic Hermitian Hamiltonian $H$. The complex eigenvalues (8) coincide with the poles of the $S$-matrix and, for small values of $\gamma$, determine energies and widths of separated resonances. With an increase of $\gamma$, the resonances start to overlap leading to specific features of the scattering process which are of our main interest.

\section{AVERAGE SCATTERING MATRIX}

The scattering matrix (14) averaged over the ensemble of decay amplitudes (3) with accuracy of $1 / N$, is given by 14 .

$$
\langle S\rangle=\frac{1-i\langle K\rangle}{1+i\langle K\rangle} .
$$

Following Refs. [14, 32], we assume, in concordance with the statistical ansatz (3), that the decay amplitudes and the eigenvalues $E_{\alpha}$ of the intrinsic Hamiltonian are statistically independent. This assumption is satisfied for the GOE and Poissonian ensembles, and here we assume that this is also true for the TBRE.

Since the mean field many-body basis $|i\rangle$ and the eigenbasis $|\alpha\rangle$ of the intrinsic Hermitian Hamiltonian are related by a real orthogonal transformation, the antiHermitian part $W$ of the effective Hamiltonian in the basis $|\alpha\rangle$ still has a factorized form in terms of the new amplitudes $B_{\alpha}^{c}$; similarly to Eq. (11), the correlation 
function of the amplitudes $B$ coincides with that given by Eq. (3). Thus, the average $K$-matrix determined by the statistical properties of the decay amplitudes reduces to

$$
\left\langle K^{a b}(E)\right\rangle=\frac{1}{2} \sum_{\alpha} \frac{\left\langle B_{\alpha}^{a} B_{\alpha}^{b}\right\rangle}{E-E_{\alpha}}=\delta^{a b} \frac{\gamma^{a}}{2 N} \sum_{\alpha} \frac{1}{E-E_{\alpha}} .
$$

The last sum in Eq.(16) is the trace of the intrinsic Green function $1 /(E-H)$. For energy $E$ inside the spectrum of $H$ we should understand it as a limiting value, $E \rightarrow E+i 0$. The trace of the imaginary part of the Green function determines the level density $\rho(E)=\sum_{\alpha} \delta\left(E-E_{\alpha}\right)$ for the Hamiltonian $H$, and

$$
\sum_{\alpha} \frac{1}{E-E_{\alpha}+i 0}=\text { P.v. } \sum_{\alpha} \frac{1}{E-E_{\alpha}}-i \pi \rho(E) .
$$

The principal value part is a smooth function of energy that vanishes in the middle of the spectrum; as a result, in this vicinity

$$
\left\langle K^{a b}\right\rangle=-i \pi \delta^{a b} \frac{\gamma^{a}}{2 N} \rho(0)=-i \delta^{a b} \kappa^{a},
$$

where $\kappa^{a}$ is defined by Eq. (44).

The average scattering matrix (15) takes the form

$$
\left\langle S^{a b}\right\rangle=\delta^{a b} \frac{1-\kappa^{a}}{1+\kappa^{a}}
$$

that depends only on our parameter of continuum coupling independently of the intrinsic interaction strength $\lambda$. This independence is due to the fact that we normalized $\kappa$ to the mean level spacing, $D$, but it is important to stress that in the shell model $D$ does depend on the intrinsic interaction [33]. Eq.(19) implies that the transmission coefficient,

$$
T^{a}=1-\left|\left\langle S^{a a}\right\rangle\right|^{2},
$$

is also independent of this strength,

$$
T^{a}=\frac{4 \kappa^{a}}{\left(1+\kappa^{a}\right)^{2}} .
$$

The transmission coefficient in the channel $a, T^{a}$, is maximum (equal to 1 ) at the critical point of this channel, $\kappa^{a}=1$, when the average $S$-matrix vanishes. Thus, $\kappa=1$ determines the so-called perfect coupling regime. We will study the statistical properties of cross sections as a function of the intrinsic interaction strength $\lambda$ and continuum coupling parameter $\kappa$, both below the critical point, $\kappa<1$, and after the superradiance transition has occurred, $\kappa>1$.

\section{COMPARING THE ENSEMBLES}

The density of states of the GOE ensemble follows the famous semicircle law, while the density of states of the
TBRE is Gaussian for large enough particle number, $n$, and orbital number, $m,[8]$; its width depends on $\lambda$. In order to compare different ensembles, we restrict our statistical analysis to a small energy interval with a constant level density at the center of the real spectrum of the complex eigenvalues of $\mathcal{H}$. This interval should be small enough in order to neglect the energy-dependent difference of the density of states among the ensembles, but large enough with respect to the widths in order to contain a statistically meaningful number of resonances.

For a model with a finite resonance number, it is important to avoid edge effects, see discussion in [9, 34]. The energy interval subject to statistical analysis should be also at a distance of at least several widths away from the edges. A rough estimate [29] goes as follows: for $M$ equivalent channels, $\Gamma / D \propto M$, and the distance from the center to the edges is $N D / 2$, then $N D / 2 \gg \Gamma / D$ that implies $M / N \ll 1 / 2$. This shows that the ratio of the number of channels to that of resonances must be small in order for the results to be model independent. With this choice, the model will be essentially equivalent to an infinite resonance model with a constant level density, apart from a narrow interval around the critical value. For $\kappa=1$, with an infinite resonance number, the average widths should logarithmically diverge, in agreement with the Moldauer-Simonius expression [11]. In our finite model, the results become model-dependent in a narrow interval near $\kappa=1$. Our approach is still appropriate for a comparison with the predictions of Ericson fluctuation theory derived for an infinite resonance model with a constant level density.

The results of numerical simulations presented below refer to the case of $N=924$ internal states and $M$ equiprobable channels, $\kappa^{a}=\kappa$. The maximum value of $M$ we considered is $M=25$ so that $M / N \approx 2 \cdot 10^{-2}$. For any value of $\kappa$, we have used a large number of realizations of the Hamiltonian matrices, with further averaging over energy.

\section{ERICSON FLUCTUATIONS}

The starting point of the conventional theory [2, 3, 4 can be summarized as follows. The scattering amplitude $\mathcal{T}^{a b}(E)=\left\langle\mathcal{T}^{a b}(E)\right\rangle+\mathcal{T}_{\mathrm{fl}}^{a b}(E)$ is divided into two parts, an average one, $\left\langle\mathcal{T}^{a b}(E)\right\rangle$, and a fluctuating one, $\mathcal{T}_{\mathrm{fl}}^{a b}(E)$, with

$$
\left\langle\mathcal{T}_{\mathrm{fl}}^{a b}(E)\right\rangle=0 .
$$

Note that in our statistical model we have $\left\langle\mathcal{T}_{\text {inel }}\right\rangle=0$ for inelastic channels, while

$$
\left\langle\mathcal{T}_{\mathrm{el}}\right\rangle=-i(1-\langle S\rangle)=-2 i \frac{\kappa}{1+\kappa}
$$

for elastic channels.

With statistical independence of poles (resonance energies) and residues (resonance amplitudes), $z_{r} \equiv \mathcal{A}_{r}^{a} \tilde{\mathcal{A}}_{r}^{b}$, 
we obtain $z_{r}=\left\langle z_{r}\right\rangle+\delta z_{r}$, so that

$$
\mathcal{T}^{a b}(E)=\sum_{r} \frac{\left\langle z_{r}\right\rangle+\delta z_{r}}{E-E_{r}+i \Gamma_{r} / 2}
$$

In the regime of overlapping resonances, $\langle\Gamma\rangle>D$, and assuming all widths of the same order, $\Gamma_{r} \sim\langle\Gamma\rangle$, the average part can be computed similarly to Eq. (17), substituting the sum by the integral,

$$
\int \frac{\rho\left(E_{r}\right)\left\langle z_{r}\right\rangle d E_{r}}{E-E_{r}+i\langle\Gamma\rangle / 2} \approx-i \frac{\pi\left\langle z_{r}\right\rangle}{D}
$$

where a constant level density, $\rho\left(E_{r}\right)=1 / D$, is assumed.

The average cross section, $\sigma=|\mathcal{T}|^{2}$, also can be divided into two contributions,

$$
\langle\sigma\rangle=\left\langle|\mathcal{T}|^{2}\right\rangle=|\langle\mathcal{T}\rangle|^{2}+\left\langle\left|\mathcal{T}_{\mathrm{ff}}\right|^{2}\right\rangle
$$

The two terms in Eq. (26) are interpreted as

$$
\langle\sigma\rangle=\left\langle\sigma_{\text {dir }}\right\rangle+\left\langle\sigma_{\mathrm{fl}}\right\rangle
$$

where the direct reaction cross section, $\left\langle\sigma_{\text {dir }}\right\rangle$, is determined by the average scattering amplitude only, while $\left\langle\sigma_{\mathrm{fl}}\right\rangle$ is the fluctuational cross section (also called in the literature the compound nucleus cross section) that is determined by the fluctuational scattering matrix.

For overlapping resonances, $\langle\Gamma\rangle>D$, the following conclusions were derived concerning the scattering amplitude and the statistical properties of the cross sections.

(A). The average fluctuational cross section [2]. Assuming that $\Gamma_{r} \approx\langle\Gamma\rangle$ for a large number of channels, i.e. fluctuations of the widths around their average value are small, $\operatorname{Var}(\Gamma) /\langle\Gamma\rangle^{2} \ll 1$, the average fluctuational cross section, $\left\langle\sigma_{\mathrm{fl}}\right\rangle=\left\langle\mathcal{T}_{\mathrm{fl}} \mathcal{T}_{\mathrm{fl}}^{*}\right\rangle$, can be written as

$$
\left\langle\sigma_{\mathrm{fl}}\right\rangle=\left\langle\sum_{r r^{\prime}} \frac{\delta z_{r}^{*} \delta z_{r^{\prime}}}{\left(E-E_{r}+i / 2\langle\Gamma\rangle\right)\left(E-E_{r}^{\prime}-i / 2\langle\Gamma\rangle\right)}\right\rangle
$$

where the substitution $\Gamma_{r} \approx\langle\Gamma\rangle$ was used. Now the averaging over energy is applied,

$$
\langle F(E)\rangle \Rightarrow \frac{1}{\Delta E} \int d E F(E) .
$$

The integration leads to

$$
\left\langle\sigma_{\mathrm{fl}}\right\rangle=\frac{2 \pi i}{\Delta E} \sum_{r r^{\prime}} \frac{\delta z_{r}^{*} \delta z_{r^{\prime}}}{E_{r^{\prime}}-E_{r}+i\langle\Gamma\rangle} .
$$

Now we assume that $\delta z_{r}$ are uncorrelated random quantities with the statistics independent of $r,\left\langle\delta z_{r}^{*} \delta z_{r^{\prime}}\right\rangle=$ $\delta_{r r^{\prime}}\left\langle|\delta z|^{2}\right\rangle$. The absence of correlations between the amplitudes $\delta z_{r}$ gives

$$
\left\langle\sigma_{\mathrm{fl}}\right\rangle=\frac{2 \pi}{D} \frac{\left\langle|\delta z|^{2}\right\rangle}{\langle\Gamma\rangle}, \quad D=\frac{\Delta E}{N} .
$$

(B). Variance of the cross section, $\operatorname{Var}(\sigma)=\left\langle\sigma^{2}\right\rangle-\langle\sigma\rangle^{2}$. The derivation can be performed under more general assumptions [35] than those used for the analysis of average cross section. If the quantities $\delta z_{r} /\left(E-\mathcal{E}_{r}\right)$ are independent complex variables, then $\mathcal{T}$ is Gaussian distributed, that is $\mathcal{T}=\xi+i \eta$, where both $\xi$ and $\eta$ are Gaussian random variables with zero mean. This is due to the fact that for $\langle\Gamma\rangle \gg D$ both $\xi$ and $\eta$ are the sums of a large number of random variables. In the conventional theory it is also assumed that $\xi$ and $\eta$ have equal variance.

Then for the fluctuating cross section we have

$$
\left\langle\sigma_{\mathrm{fl}}^{2}\right\rangle=\left\langle\left|\mathcal{T}_{\mathrm{fl}}\right|^{4}\right\rangle=\left\langle\mathcal{T}_{\mathrm{fl}} \mathcal{T}_{\mathrm{fl}}^{*} \mathcal{T}_{\mathrm{fl}} \mathcal{T}_{\mathrm{fl}}^{*}\right\rangle=2\left\langle\sigma_{\mathrm{fl}}\right\rangle^{2}
$$

In a more general case when $\langle\mathcal{T}\rangle \neq 0$,

$$
\operatorname{Var}(\sigma)=\left\langle\sigma_{\mathrm{fl}}\right\rangle\left(2\left\langle\sigma_{\mathrm{dir}}\right\rangle+\left\langle\sigma_{\mathrm{f}}\right\rangle\right)
$$

(C). The correlation function of the scattering amplitudes is defined as

$c(\epsilon)=\left\langle\mathcal{T}(E+\epsilon) \mathcal{T}^{*}(E)\right\rangle-|\langle\mathcal{T}(E)\rangle|^{2}=\left\langle\mathcal{T}_{\mathrm{fl}}(E+\epsilon) \mathcal{T}_{\mathrm{fl}}^{*}(E)\right\rangle$.

Evaluating $c(\epsilon)$ under the same assumptions as for the average cross sections, one obtains,

$$
c(\epsilon)=\left\langle\sigma_{\mathrm{fl}}\right\rangle \frac{\langle\Gamma\rangle}{\epsilon+i\langle\Gamma\rangle} .
$$

(D). The cross section correlation function is defined as

$$
C(\epsilon)=\langle\sigma(E) \sigma(E+\epsilon)\rangle-\langle\sigma(E)\rangle^{2}
$$

Taking into account the Gaussian form of distribution for $\mathcal{T}$ and Eq.(35), one obtains that:

(a) the normalized autocorrelation function of cross sections satisfies the relation,

$$
\frac{C(\epsilon)}{C(0)}=\frac{|c(\epsilon)|^{2}}{|c(0)|^{2}}
$$

(b) the correlation function has a Lorentzian form,

$$
\frac{C(\epsilon)}{C(0)}=\frac{l^{2}}{l^{2}+\epsilon^{2}},
$$

where the correlation length, $l$, is equal to the average width,

$$
l=\langle\Gamma\rangle
$$

In the following we compare the predictions (B)-(D) of the conventional theory of Ericson fluctuations with our numerical results, paying special attention to the dependence on the intrinsic interaction strength, $\lambda$. As for the part $(\mathrm{A})$, since there are no predictions for the quantity $|\delta z|^{2}$ determining the average fluctuational cross section (31), the comparison will be done with the HauserFeshbach, theory widely used in the literature. 


\section{AVERAGE CROSS SECTION}

In this section we study how total and partial cross sections depend on the continuum coupling, $\kappa$, and intrinsic interaction, $\lambda$. For any value of $\kappa$ we have used $N_{r}=30$ realizations of the Hamiltonian matrices. For each realization we took into account only the interval $[-0.2,0.2]$ of real energy at the center of the spectrum.

It follows from Eq. (14) that the average total cross section defined by the optical theorem,

$$
\left\langle\sigma_{\text {tot }}\right\rangle=2(1-\operatorname{Re}\langle S\rangle)=\frac{4 \kappa}{1+\kappa},
$$

depends only on the average scattering matrix and therefore is independent of $\lambda$ and $M$. Since $\sigma_{\mathrm{el}}=\sigma_{\mathrm{tot}}$ for $M=1$, the average elastic cross section is also independent of $\lambda$ for the case of one channel. The situation changes as we increase the number of channels.

In order to analyze the average elastic and inelastic cross section, we single out the average scattering matrix elements in the standard form, $S^{a b}=\left\langle S^{a b}\right\rangle+S_{\mathrm{fl}}^{a b}$, where $\left\langle S^{a b}\right\rangle=\delta^{a b}\left\langle S^{a a}\right\rangle$ and $\left\langle S_{\mathrm{fl}}^{a b}\right\rangle=0$. The average inelastic cross section, $a \neq b$, is given by

$$
\left\langle\sigma^{\mathrm{ab}}\right\rangle=\left\langle\left|S^{a b}\right|^{2}\right\rangle=\left\langle\left|S_{\mathrm{fl}}^{a b}\right|^{2}\right\rangle
$$

The average elastic cross section can be written as

$$
\left\langle\sigma^{a a}\right\rangle=\left|1-\left\langle S^{a a}\right\rangle\right|^{2}+\left\langle\left|S_{\mathrm{fl}}^{a a}\right|^{2}\right\rangle .
$$

Following the literature we will call $\left\langle\left|S_{\mathrm{fl}}^{a b}\right|^{2}\right\rangle$ the fluctuational cross section, $\left\langle\sigma_{\mathrm{ff}}^{a b}\right\rangle$. For $M$ equivalent channels the fluctuational cross section can be expressed with the use of the elastic enhancement factor,

$$
F=\frac{\left\langle\sigma_{\mathrm{fl}}^{a a}\right\rangle}{\left\langle\sigma_{\mathrm{fl}}^{a b}\right\rangle}
$$

where $b \neq a$. Indeed, in the case of equal channels, using the relation,

$$
\sigma_{\mathrm{tot}}=\sum_{b} \sigma^{a b}=(M-1) \sigma^{\mathrm{inel}}+\left|1-S^{a a}\right|^{2},
$$

we obtain

$$
\left\langle\sigma_{\mathrm{fl}}^{a b}\right\rangle=\frac{1-\left|\left\langle S^{a a}\right\rangle\right|^{2}}{F+M-1}=\frac{T}{F+M-1},
$$

where $T$ is the transmission coefficient defined in Eq. (21), and

$$
\left\langle\sigma_{\mathrm{fl}}^{a a}\right\rangle=F\left\langle\sigma_{\mathrm{fl}}^{a b}\right\rangle=\frac{F T}{F+M-1} ; \quad a \neq b .
$$

Since the transmission coefficient $T$ does not depend on $\lambda$, the only dependence on $\lambda$ in Eqs. (45) and (46) is contained in the elastic enhancement factor $F$. The same seems to be correct even when the channels are nonequivalent, according to the results of Ref. [36]. Leaving a detailed analysis of the elastic enhancement factor for a separate study, here we point out that $F$ also depends on $\kappa$. Specifically, with an increase of $\kappa$ from zero, the value of $F$ decreases, being confined by the interval between 3 and 2 .

For the fluctuational inelastic cross section, with an increase of the number of channels the dependence on the interaction strength $\lambda$ disappears, see Fig. 1 (upper panel). This is in agreement with the fact that in the limit of large $M$ we have $\left\langle\sigma_{\mathrm{fl}}^{a b}\right\rangle \rightarrow T / M$, independently of $\lambda$, see Eq. (45). In contrast, the fluctuational elastic cross section manifests a clear dependence on $\lambda$, see Fig. 1 (lower panel). Thus, one can directly relate the value of the enhancement factor $F$ to the strength $\lambda$ of interaction between the particles. As one can see, the more regular is the intrinsic motion, the higher is the average cross section. Note that for a large number of channels the $\lambda$-dependence of the elastic cross section is in agreement with the estimate, $\left\langle\sigma_{\mathrm{fl}}^{a a}\right\rangle \rightarrow F T / M$, see Eq. (46).
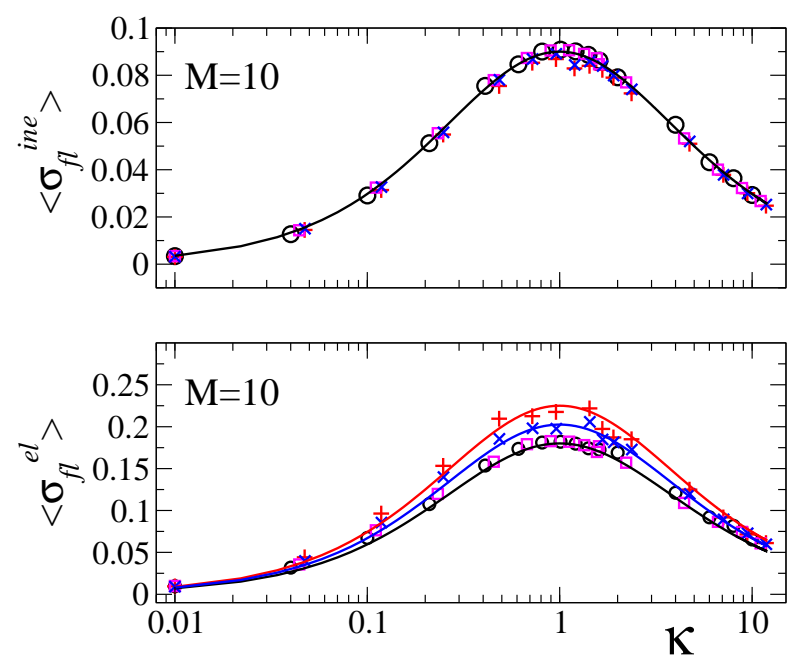

FIG. 1: (Color online) Fluctuational inelastic (upper panel) and fluctuational elastic (lower panel) cross sections as a function of $\kappa$. Circles refer to the GOE case, pluses to $\lambda=0$, crosses to $\lambda=1 / 30$, and squares to $\lambda \rightarrow \infty$. The solid curves (top-down) correspond to the HF formula (49) with $F=2.5,2.25,2.0$ for $\lambda=0,1 / 30$ and the GOE, respectively. The values 2.5 and 2.25 are found numerically using the definition (43).

Now we compare our numerical results with the Hauser-Feshbach (HF) formula derived under quite general assumptions for both $a=b$ and $a \neq b$ (see Ref. [8] and references therein),

$$
\left\langle\sigma_{\mathrm{fl}}^{a b}\right\rangle=\left(1+\delta_{a b}\right) \frac{T^{a} T^{b}}{\sum T^{c}}=\left(1+\delta_{a b}\right) \frac{T}{M} .
$$

Here the last expression corresponds to our case of equivalent channels, $T^{c}=T$. As one can see, the HF formula predicts $F=2$, independently of the interaction strength 
between the particles. This formula was also derived in [6] in the overlapping regime for the TBRE ensemble with the infinite interaction, and in [37] for the GOE ensemble. For finite number of channels in the limit $1 / M \ll 1$, the corrected HF formula was derived in [6]. For equivalent channels it reads,

$$
\left\langle\sigma_{\mathrm{fl}}^{a b}\right\rangle=\left(1+\delta_{a b}\right) \frac{T}{M}\left(1-\frac{1}{M}\right) .
$$

Our data confirm that for the fluctuational inelastic cross section the HF formula gives correct results for all values of $\lambda$ in the case of large number of channels. The specific case of small number of channels, for which the $\mathrm{HF}$ is not valid, will be discussed elsewhere.

On the other hand, for the fluctuational elastic cross section, our data show that the HF formula works only in the GOE case and in the limit $\lambda \rightarrow \infty$, see Fig. 1 (lower panel). At finite values of $\lambda$ clear deviations are seen. In order to describe the data, we modified the HF formula taking into account that the elastic enhancement factor varies with $\lambda$,

$$
\left\langle\sigma_{\mathrm{fl}}^{a b}\right\rangle=\left[1+\delta^{a b}(F-1)\right] \frac{T}{M}\left(1-\frac{1}{M}\right) .
$$

As one can see, this expression gives a satisfactory description of the data, with the numerically computed values of $F$. The problem of an analytical dependence of $F$ on the interaction strength $\lambda$ remains open. To shed light on this problem, we performed a specific study of the elastic cross section in dependence on $\lambda$ for fixed value $\kappa=0.8$ in the overlapping regime, see Fig. 2. As one see, there is a sharp decrease of the cross section in the transition from regular to chaotic intrinsic motion, $\lambda \approx \lambda_{\text {cr }}$. This result is quite instructive since it shows how the scattering properties are influenced by the onset of chaos in an internal dynamics. The non-trivial point is that the analytical estimate of $\lambda_{\text {cr }}$ was obtained for a closed system, $\kappa=0$. However, even in the regime of a strong coupling to the continuum, $\kappa=0.8$, this estimate gives a correct value for the interaction strength at which a drastic change of scattering properties occurs.

\section{FLUCTUATIONS OF WIDTHS AND RESONANCE AMPLITUDES}

Here we discuss the conventional assumption that for a large number of channels the deviations of the widths from their average are small, $\operatorname{Var}(\Gamma) /\langle\Gamma\rangle^{2} \ll 1$, and, therefore, for analytical estimates one can set $\Gamma_{r} \approx\langle\Gamma\rangle$, see Eq. (28). It is usually said in justification of this assumption [2] that in the overlapping regime the width can be presented as a sum of partial widths, $\Gamma_{r}=\sum_{c=1}^{M} \Gamma_{r}^{c}$. Assuming that individual partial widths obey the PotterThomas distribution, the total width is expected to have a $\chi_{M}^{2}$ distribution, so that $\operatorname{Var}(\Gamma) /\langle\Gamma\rangle^{2}=2 / M$ is small for $M \gg 1$. In fact, it is sufficient to accept that the partial widths are independent random variables; then $\operatorname{Var}(\Gamma) \propto M$ and $\langle\Gamma\rangle \propto M$, so that $\operatorname{Var}(\Gamma) /\langle\Gamma\rangle^{2} \propto M^{-1}$.

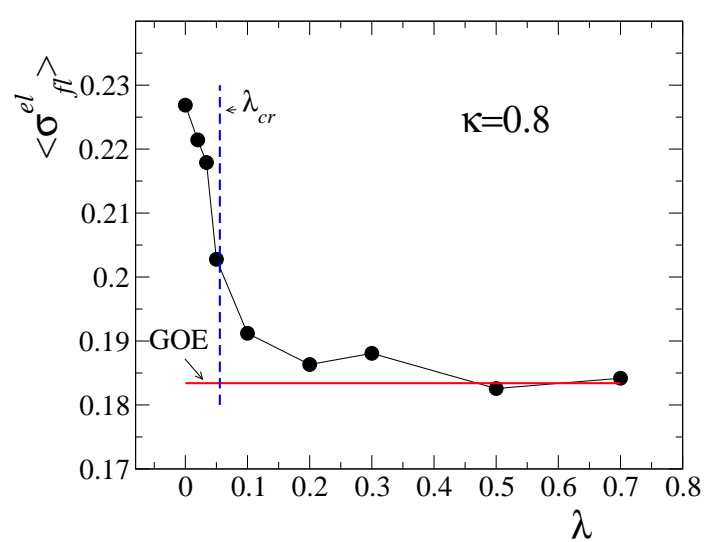

FIG. 2: (Color online) Fluctuational elastic cross section as a function of the interaction strength $\lambda$ for $M=10$ and $\kappa=0.8$ (connected circles). The horizontal line refers to the GOE value, and dashed vertical line shows the critical value $\lambda_{\mathrm{cr}}$ for the transition to chaos in the TBRE, see Eq. (2).

However, in our previous work 29] we have showed that for large values of $\kappa$ the distribution of the widths strongly differs from the $\chi_{M}^{2}$ distribution. Our new data in Fig. 3 give more details concerning this problem. These data were obtained for a large number $N_{r}=100$ realizations of the Hamiltonian matrices, in order to have reliable results.

The data show that as $\kappa$ increases the normalized variance, $\operatorname{Var}(\Gamma) /\langle\Gamma\rangle^{2}$, also increases, remaining very large even for $M=20$. Moreover, the deviations from the expected $1 / M$ behavior are clearly seen signaling the presence of correlations in the partial widths. From Fig. 3 one can also understand how the value of $\operatorname{Var}(\Gamma) /\langle\Gamma\rangle^{2}$ depends on the degree of intrinsic chaos determined by the parameter $\lambda$. Specifically, for small $\kappa$ there is no dependence on $\lambda$ and $\operatorname{Var}(\Gamma) /\langle\Gamma\rangle^{2}$ decreases as $2 / M$ for all the ensembles, as expected. However, as $\kappa$ grows, the dependence on $\lambda$ emerges: the weaker the intrinsic chaos (and, consequently, the more ordered is the intrinsic spectrum) the larger are the width fluctuations.

In Ref. 23] the widths distribution for the GOE ensemble was found analytically for any number of channels in the limit of $N \rightarrow \infty$ and $M$ fixed. The general analytical result is given in terms of a complicated threefold integral. A simpler expression is obtained for a specific case $M=2$, for which the distribution of the widths is expressed as a double integral,

$$
\left\langle y^{2}\right\rangle=C \int_{1}^{g} \frac{d \nu}{\sqrt{\nu^{2}-1}} \int_{-1}^{1} d \mu \frac{\left(1-\mu^{2}\right)}{(\nu+g-2 \mu)(\nu-\mu)^{2}},
$$

where $C=1 /\left(2 \sqrt{g^{2}-1}\right), y=\pi \Gamma / D, g=(2 / T)-1$ and $T$ is the transmission coefficient, so that from Eq. (21) we have $g=\left(1+\kappa^{2}\right) / 2 \kappa$, or $\kappa=g \pm \sqrt{g^{2}-1}$. Note that 


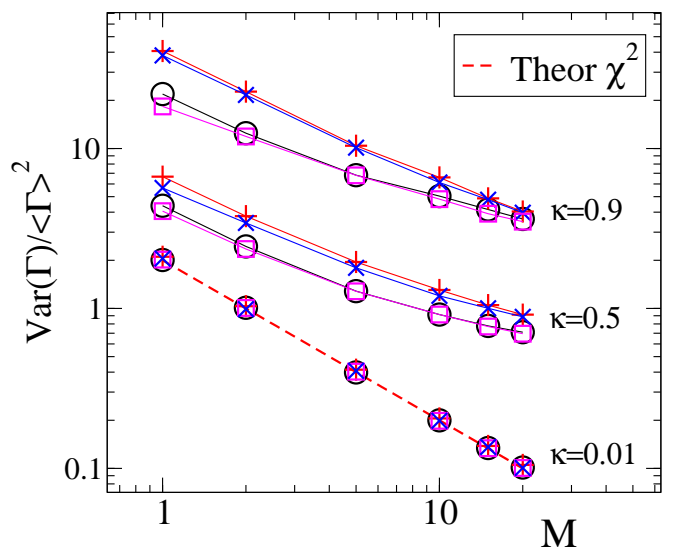

FIG. 3: (Color online) Normalized variance of the width as a function of the number of channels $M$, for different coupling strengths $\kappa$ (connected symbols are the same as in Fig. 1). While for small coupling, $\kappa=0.01$, the variance decreases with the number of channels very fast in accordance with the expected $\chi^{2}$-distribution (dashed line), for large couplings, $\kappa=0.5$ and 0.9 , the behavior is different from the $1 / M$ dependence.

$\nu+g-2 \mu>0$ and for $g=1$ we have $\kappa=1$. The result of numerical integration in Eq. (50) is shown in Fig. (4 by a solid curve. The comparison with the normalized variance of the widths for the GOE case and $M=2$ (circles) shows a good agreement except for the vicinity

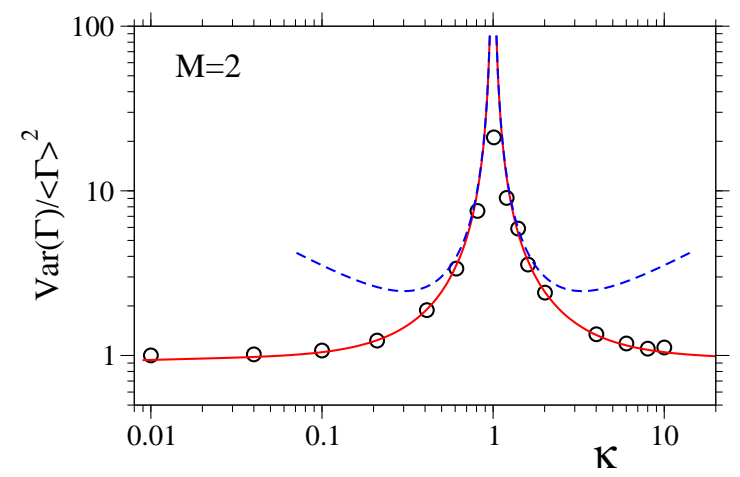

FIG. 4: (Color online) Numerical data for the normalized variance of the widths vs $\kappa$ for GOE and $M=2$ (circles), in comparison with the result of numerical integration of Eq. (50) (solid curve), and with Eq. (51) (dashed curve), see in the text.

A specific interest is in the behavior of the variance at the transition region, $\kappa \approx 1$. The analytical expression, derived from Eq. (50) for this region has the form,

$$
\frac{\operatorname{Var}(\Gamma)}{\langle\Gamma\rangle^{2}}=\frac{2(2+\pi)}{\sqrt{2(g+1)}(g-1)}\left(\ln \frac{g-1}{g+1}\right)^{2}-1,
$$

and is shown in Fig. 4 by the dashed curve. From the above relation for $M=2$ one can obtain that the normalized variance diverges as

$$
\frac{\operatorname{Var}(\Gamma)}{\langle\Gamma\rangle^{2}} \propto \frac{1}{(1-\kappa)^{2}}[\ln (1-\kappa)]^{2} .
$$

Our numerical simulations confirm that the divergence remains for any number of channels and for any value of $\lambda$. Thus, contrary to the traditional belief, the variance of widths does not become small for a large number of channels. Another result is that the assumption of the absence of correlations between the resonance amplitudes $\delta z_{r}$ and the widths $\Gamma_{r}$ seems to be incorrect in the region of a strong resonance overlap. Indeed, the data reported in Fig. 5 demonstrate that in contrast with the case of weak coupling, $\kappa=0.001$, for a strong coupling there are systematic correlations between $\delta z_{r}$ and $\Gamma_{r}$. These correlations are increasing with an increase of the cou-

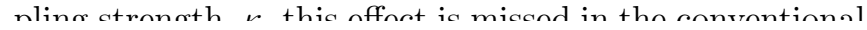

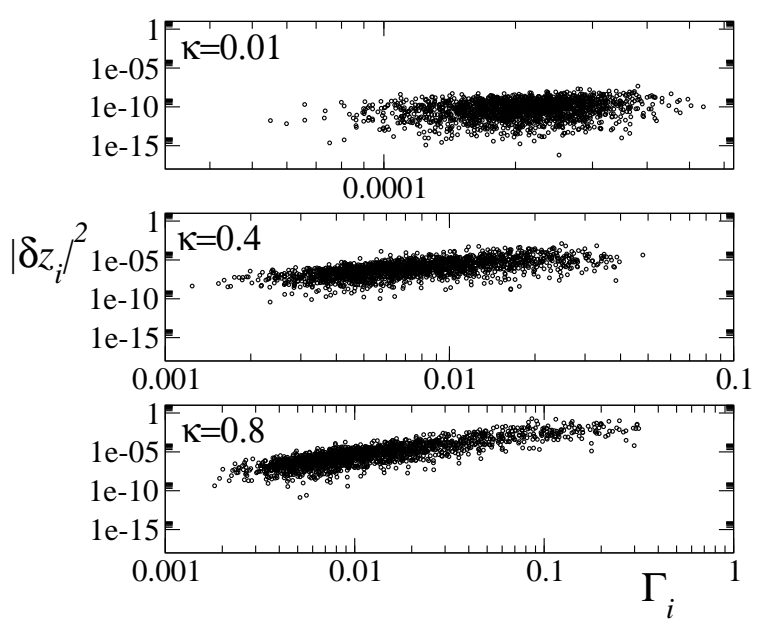

FIG. 5: (Color online) Absolute squares of resonance amplitudes, $\left|\delta z_{r}\right|^{2}$, versus the widths $\Gamma_{r}$ for the GOE with $M=20$. As $\kappa$ increases, the correlations between $\left|\delta z_{r}\right|^{2}$ and $\Gamma_{r}$ grow.

\section{STATISTICS OF CROSS SECTIONS}

\section{A. Distribution of fluctuational cross sections}

According to the standard Ericson theory, the fluctuating scattering amplitude can be written as $\mathcal{T}_{\mathrm{fl}}^{a b}=\eta+i \xi$, where $\eta$ and $\xi$ are Gaussian random variables with zero mean and equal variances. Since the fluctuating cross 
section is given by

$$
\sigma_{\mathrm{fl}}=\left|\mathcal{T}_{\mathrm{ff}}\right|^{2}=|\eta|^{2}+|\xi|^{2},
$$

then $\sigma_{\mathrm{fl}}$ should have a $\chi^{2}$ distribution with two degrees of freedom, that is an exponential distribution,

$$
P(x)=e^{-x}, \quad x=\frac{\sigma_{\mathrm{fl}}}{\left\langle\sigma_{\mathrm{fl}}\right\rangle} .
$$

This should be valid both for the elastic and inelastic cross sections.

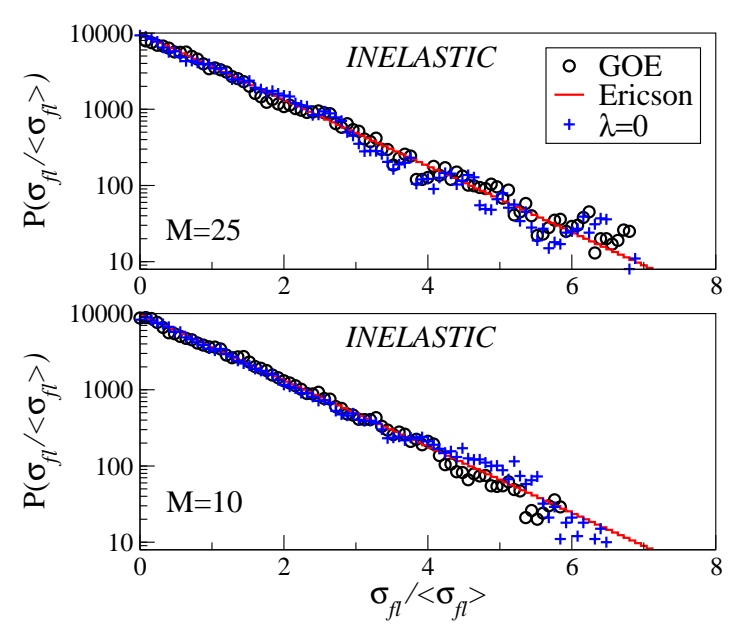

FIG. 6: (Color online) Distribution of the inelastic fluctuational cross section for the GOE and for the $\lambda=0$ case, for $M=10 ; 25$ number of channels and fixed $\kappa=0.9$.

In Figs. 6 and 7 we show the distribution of fluctuational cross sections for the elastic and inelastic cross sections, with two different numbers of channels, $M=10$ and $M=25$. Analyzing these data, one can draw the following conclusions. First, for the inelastic cross section the data seem to follow the predicted exponential distribution. It should be noted, however, that a more detailed analysis with the help of the $\chi^{2}$-test reveals the presence of strong deviations.

The situation with the fluctuational elastic cross section is different due to strong deviations from the exponential distribution occurring even for a quite large $M=25$. The fact that large deviations from the conventional theory (for finite values of $M$ ) should be expected in the elastic case were recognized also in Refs. [38, 39]. The comparison between $M=10$ and $M=25$ cases indicate that it is natural to assume that with a further increase of $M$ both the distributions will converge to the exponential one. It is important to note that there is a weak dependence on the interaction strength $\lambda$ between the particles. This is confirmed by a closer inspection of the data of Fig. 7 Specifically, the data clearly show that there is a systematic difference for the two limiting

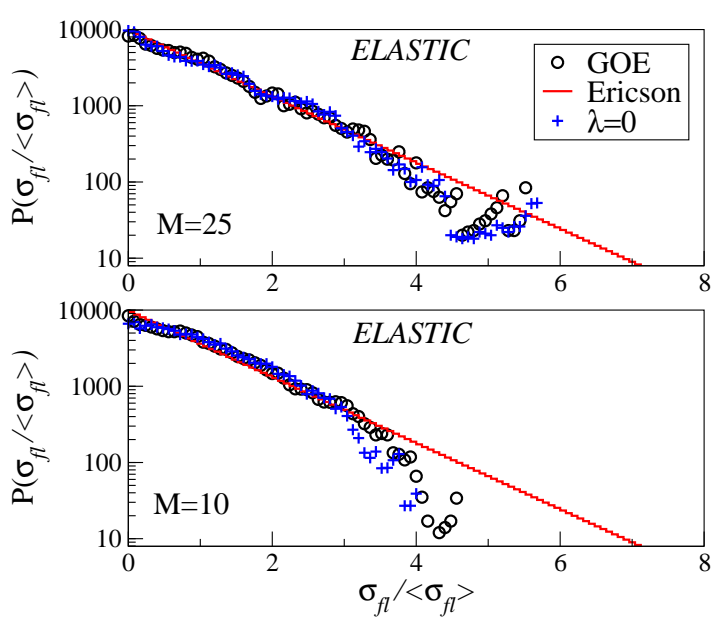

FIG. 7: (Color online) The same as Fig.6, but for the elastic fluctuational cross section. A clear difference from the exponential distribution is seen in the tails.

cases of zero and infinitely large values of $\lambda$. Our results for the normalized variance (see below), indeed, confirm a presence of this weak dependence on $\lambda$.

The above data for the distribution of the normalized fluctuational cross section may be treated as a kind of confirmation of the Ericson fluctuation theory. However, it should be stressed that if we are interested in the fluctuations of non-normalized cross sections (at least, for elastic cross sections), one should take into account the dependence on $\lambda$. Currently no theory allows one to obtain the corresponding analytical results, even for the situation where the number of channels is sufficiently large.

\section{B. Fluctuations}

Here we compare our results for the variance of cross sections with the Ericson fluctuations theory [2], and with more recent results for the GOE [38, 39]. According to the standard predictions, the variance of fluctuations of both elastic and inelastic cross sections,

$$
\operatorname{Var}\left(\sigma^{a b}\right)=\left\langle\left(\sigma^{a b}-\left\langle\sigma^{a b}\right\rangle\right)^{2}\right\rangle,
$$

is directly connected to the average cross sections by Eq. (33). It is useful to express the variance of the cross sections in terms of the scattering matrix. In our statistical model for $a \neq b,\left\langle\mathcal{T}^{a b}\right\rangle=i\left\langle S^{a b}\right\rangle=0$. Therefore, the variance of the inelastic cross section reads

$$
\operatorname{Var}\left(\sigma^{a b}\right)=\left\langle\sigma_{\mathrm{fl}}^{a b}\right\rangle^{2}=\left\langle\left|S_{\mathrm{fl}}^{a b}\right|^{2}\right\rangle^{2} .
$$

For the elastic scattering one can write $\left\langle\mathcal{T}^{a a}\right\rangle=-i(1-$ $\left.\left\langle S^{a a}\right\rangle\right)$, so that $\sigma_{\mathrm{dir}}=\left|1-\left\langle S^{a a}\right\rangle\right|^{2}$, and $\sigma_{\mathrm{fl}}=\left\langle\left|S_{\mathrm{fl}}^{a a}\right|^{2}\right\rangle$. 
Therefore, for the variance of the elastic cross sections, one obtains,

$$
\begin{aligned}
& \operatorname{Var}\left(\sigma^{a a}\right)=2\left\langle\sigma_{\mathrm{fl}}^{a a}\right\rangle\left\langle\sigma_{\mathrm{dir}}^{a a}\right\rangle+\left\langle\sigma_{\mathrm{fl}}^{a a}\right\rangle^{2}= \\
& =\left\langle\left|S_{\mathrm{fl}}^{a a}\right|^{2}\right\rangle^{2}+2\left|1-\left\langle S^{a a}\right\rangle\right|^{2}\left\langle\left|S_{\mathrm{fl}}^{a a}\right|^{2}\right\rangle,
\end{aligned}
$$
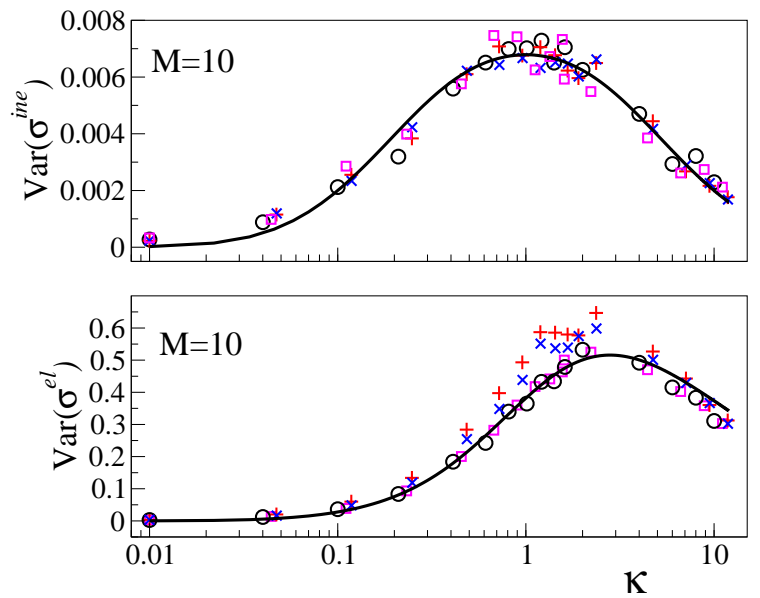

FIG. 8: (Color online) Variance of inelastic (upper panel) and elastic (lower panel) cross sections, see Eq. (27), for $M=10$ as a function of $\kappa$ for different interaction strengths: the GOE (circles), $\lambda=0$ (pluses), $\lambda \rightarrow \infty$ (squares), and $\lambda=1 / 30$ (crosses). For the comparison the theoretical curve for $M T \gg$ 1, obtained for the GOE from Eqs. (60]61) (solid curve) is also added. Note that the maximum of $\operatorname{Var}\left(\sigma^{e l}\right)$ is shifted from $\kappa=1$ due to the presence of direct processes.

The discussed above conventional predictions and our analysis of the average cross section in Sec. VI imply that the variances of cross sections depend on the intrinsic interaction strength $\lambda$ through the average cross sections. Our data for a relatively large number $M=10$ of channels, see Fig. 8, indeed, correspond to this expectation. Since for the inelastic scattering the average cross section does not depend on the interaction strength, it is quite expected that the same occurs for the variance of the inelastic cross section. Our data confirm this expectation. On the other hand, the variance of the elastic cross section reveals a clear dependence on the value of $\lambda$. As one can see, this dependence is quite strong in the region of strongly overlapped resonances, for $\kappa \approx 1$.

Let us now compare our data with the exact expressions for the variance of cross sections. To do this, it is convenient to express this variance in terms of the scattering matrix,

$$
\operatorname{Var}\left(\sigma^{a b}\right)=\left\langle\left|S_{\mathrm{fl}}^{a b}\right|^{4}\right\rangle-\left\langle\left|S_{\mathrm{fl}}^{a b}\right|^{2}\right\rangle^{2}+
$$

$-\delta^{a b}\left(2\left[\left(1-\left\langle S^{a a *}\right\rangle\right)\left\langle\left|S_{\mathrm{fl}}^{a a}\right|^{2} S_{\mathrm{fl}}^{a a}\right\rangle+\right.\right.$ c.c. $\left.]-2\left|1-\left\langle S^{a a}\right\rangle\right|^{2}\left\langle\left|S_{\mathrm{fl}}^{a b}\right|^{2}\right\rangle\right)$
Comparing Eq. (58) with the standard predictions, Eqs. (56 57), one can see that they are correct if:

$$
\begin{gathered}
\text { (i) }\left\langle\left|S_{\mathrm{fl}}^{a b}\right|^{4}\right\rangle-2\left\langle\left|S_{\mathrm{fl}}^{a b}\right|^{2}\right\rangle^{2}=0, \\
\text { (ii) }\left\langle S_{\mathrm{fl}}^{a a}\left|S_{\mathrm{fl}}^{a b}\right|^{2}\right\rangle=0 .
\end{gathered}
$$

These properties are consistent with the Gaussian character of the distribution for the fluctuational scattering matrix.

The analytical expressions for the variance of elastic and inelastic cross sections were obtained in Refs. [38, 39] for the GOE case, any number of channels and any coupling strength with the continuum. However, simple expressions were derived only for $M T \gg 1$. Even under such a condition, the analytical results show deviations from the conventional assumptions. Specifically, it was found,

$$
\begin{gathered}
\text { (i) }\left\langle\left|S_{\mathrm{fl}}^{a b}\right|^{4}\right\rangle-2\left\langle\left|S_{\mathrm{fl}}^{a b}\right|^{2}\right\rangle^{2}= \\
=\left(1+7 \delta^{a b}\right)\left[6-4\left(T^{a}+T^{b}\right)+r_{2}\right] \frac{2\left(T^{a} T^{b}\right)^{2}}{\left(S_{1}+1\right)^{3}}
\end{gathered}
$$

and

$$
\text { (ii) }\left\langle\left|S_{\mathrm{fl}}^{a a}\right|^{2} S_{\mathrm{fl}}^{a a}\right\rangle=-8\left\langle S^{a a *}\right\rangle \frac{\left(T^{a}\right)^{3}}{\left(S_{1}+1\right)^{2}},
$$

where $S_{1}=\sum T^{c}, S_{2}=\sum\left(T^{c}\right)^{2}$ and $r_{2}=\left(S_{2}+1\right) /\left(S_{1}+\right.$ 1).

The theoretical values for the variance of the cross sections obtained from Eqs.6061) and from the HF formula, through Eq.(58), are shown in Fig. 8 by solid curve. The agreement is good for the GOE case in the strong coupling regime, as expected. As we can see from Eqs. (6061), assumptions of Eq. (59) are valid for large $S_{1}$. In particular it was shown in [38, 39] that the ratio $\operatorname{Var}\left(\sigma_{\mathrm{fl}}\right) /\left\langle\sigma_{\mathrm{fl}}\right\rangle^{2}$, being equal to one in standard theory, significantly differs from unity in the range $10<M T<20$, where this theory is expected to be valid.

It is now instructive to see how the normalized variance of the cross sections depends on the number of channels, see Fig. 9. According to the Ericson prediction, the ratio of the variance to the square of the mean of the cross sections has to be 1 for strongly overlapped resonances, in the limit of large number of channels. As one can see, the data for the inelastic scattering roughly confirm this prediction. It is not a surprise that practically there is no dependence on the strength $\lambda$ of interaction between the particles. On the other hand, there is a small systematic difference from the predicted value, that emerges for all values of $\lambda$, as well as for the GOE case. One can expect that this difference disappears for a much larger number of channels.

A more interesting result arises for the elastic cross - section. As one can see from Fig. 9] in this case there is a strong difference from the limiting value of one even in 

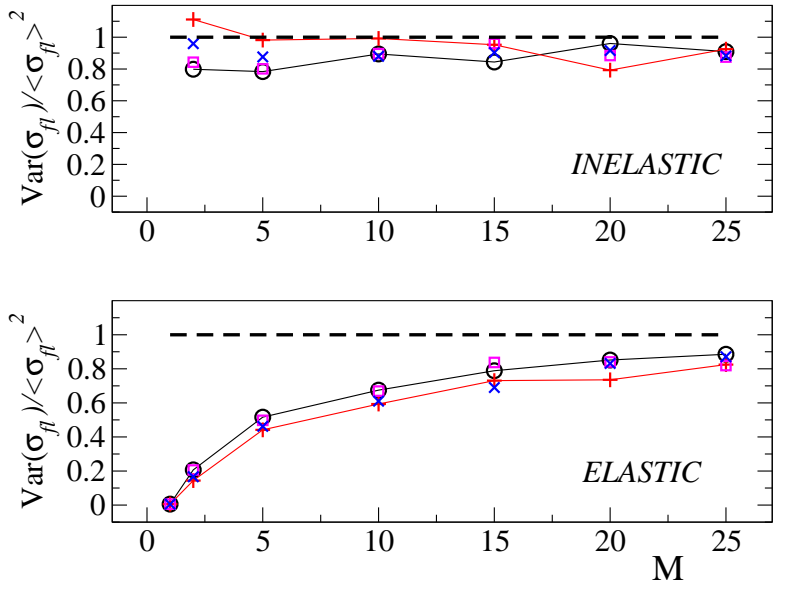

FIG. 9: (Color online) Normalized variance for $\kappa=0.9$ versus the number of channels for the elastic and inelastic cross sections: GOE case (connected circles), $\lambda=0$ (connected pluses), $\lambda \rightarrow \infty$ (squares), and $\lambda=1 / 30$ (crosses). The theoretical value in the Ericson theory, $\operatorname{Var}(\sigma) /\langle\sigma\rangle^{2}=1$, is shown as a dashed line.

the case when the number of channels is relatively large, $M=10-20$. The data clearly indicate that for the applicability of the standard theory one needs to have a very large number of channels, at least larger than $M=$ 25.

Another instructive observation is a weak dependence of the normalized variance on the interaction strength $\lambda$. This result is in agreement with the data reported in Fig. 7 for the distribution of individual values of the cross section, where a systematic deviation can be seen when comparing the GOE case with the case of $\lambda=0$. We would like to stress that the weak $\lambda$-dependence is in contrast with a strong dependence occurring for the nonnormalized variance, see Fig. 8. One can treat this effect as manifesting that both the variance and the square of the cross section average depend on $\lambda$ practically in the same way. Therefore, their ratio turns out to be almost independent on $\lambda$. As one can see, although the standard predictions are not correct for the non-normalized variance, they are in a good correspondence with the data for the normalized variance.

\section{Correlation functions}

Here we compare our results for the correlation function of cross sections and the scattering matrix with the standard predictions, see Section V. The correlation functions for the cross section and for the scattering matrix were computed according to Eqs. (34/36) for the elastic and inelastic cross sections. The correlation lengths for the cross section, $l_{\sigma}$, and for the scattering matrix, $l_{S}$, are defined as the energy for which the correlation function is $1 / 2$ of its initial value. Our results can be summarized as follows.

(A) For large $M$, we found $l_{\sigma} \approx l_{S}$ for any interaction strength $\lambda$. On the contrary, for smaller $M$, our data show that $l_{S}<l_{\sigma}$, and this difference grows for the weaker interaction between the particles, $\lambda$.

(B) For large $M$, the correlation functions are Lorentzian for all $\lambda$, while for a small number of channels the correlation function is not Lorentzian, in agreement with the results of [40]. Moreover, for any $M$, the correlation length is different from the average width, as one can expect due to Eq. (39), apart from the region of small $\kappa$, see Fig. 10. For a large number of channels, the correlation length is determined instead by the transmission coefficient through the Weisskopf relation, see [37] and references therein,

$$
\frac{l}{\Gamma}=\frac{M T}{9 \cdot \pi}=\frac{M}{9 \cdot \pi} \frac{4 \kappa}{(1+\kappa)^{2}}
$$
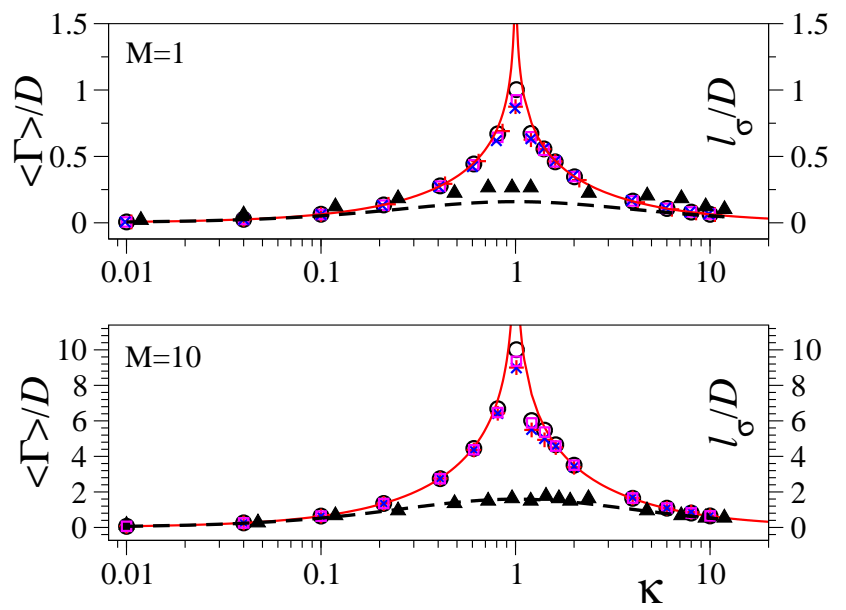

FIG. 10: (Color online) Average width, $\langle\Gamma\rangle$, and correlation length, $l_{\sigma}$, normalized to the mean level spacing at the center of the spectra, versus $\kappa$ for $M=1$ (upper panel) and $M=10$ (lower panel). Solid curves show the MS-expression (61). Open circles refer to the GOE case, pluses to $\lambda=0$, squares to $\lambda \rightarrow \infty$, and crosses to $\lambda=1 / 30$, all for the normalized average width. Full triangles stand for the normalized correlation length at $\lambda=1 / 30$. The dashed line shows the Weisskopf relation (60).

In Fig. 11, it is shown that, for a large number of channels, the elastic correlation length is in agreement with Eq.62) for all values of the interaction strength, $\lambda$. The same occurs for the inelastic correlation length.

The Weisskopf relation (62) has been also derived in Ref. 6] for small values of the ratio $m=M / N$, in the overlapping regime for the TBRE ensemble with the infinite interaction, as well as in Ref. 7] for the GOE ensemble. In [37] the correlation function for the GOE ensemble was computed also when $m=M / N$ is not small, and the deviations from Eq. (62) and from the Lorentzian form of the correlation function were found. This is not in contrast with our results for the case of small ratio of $m$, see discussion in Sec. IV. 

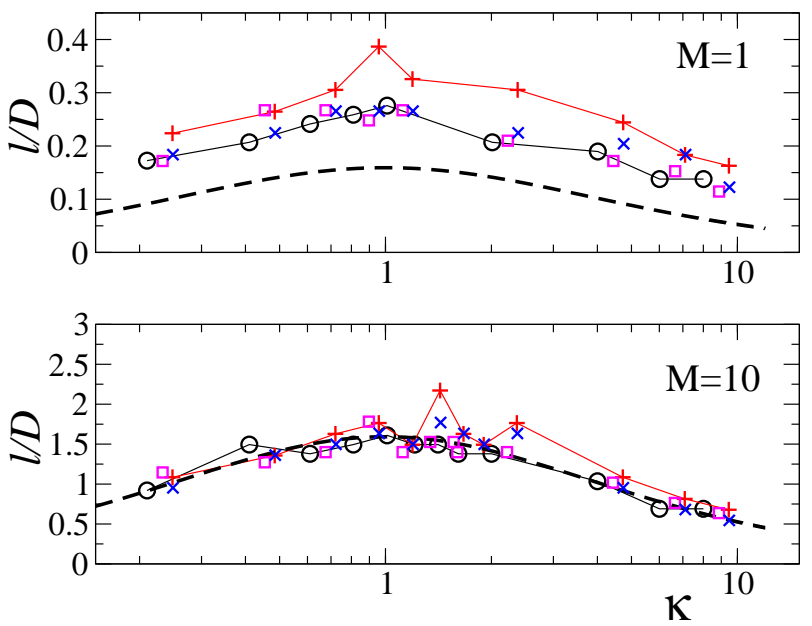

FIG. 11: (Color online) Elastic-elastic correlation length of the cross section at different values of $\lambda$ as a function of $\kappa$ for $M=1$ and $M=10$; the GOE case (connected circles), $\lambda=0$ (pluses connected by a line), $\lambda \rightarrow \infty$ (squares), and $\lambda=1 / 30$ (crosses). The Weisskopf relation (62) is shown by dashed curve.

The fact that the correlation length is not equal to the average width was recognized long ago, see Refs. 34] and [8]. However, the statements based on the equality (39) still appear in the literature, see, for example, Ref. [41]. The relation between the average resonance width and the transmission coefficient is given by the MoldauerSimonius (MS) formula [9, 11],

$$
M \ln (1-T)=-2 \pi \frac{\langle\Gamma\rangle}{D} .
$$

It can be seen from this expression and Eq. (62) that the equality $l=\langle\Gamma\rangle$ is true only for small $T$.

\section{CONCLUSIONS}

In conclusion, we have studied the statistics of cross sections for a fermion system coupled to open decay channels. For the first time we carefully followed various signatures of the crossover from isolated to overlapping resonances in dependence on the strength of inter-particle interaction modelled here by the two-body random ensemble. The study was performed for the simplest Gaussian ensemble of decay amplitudes. Even in this limiting case, when these amplitudes were considered as uncorrelated with the intrinsic dynamics, we found significant dependence of reaction observables on the strength of intrinsic interaction. We expect this dependence to be amplified with realistic interplay of decay amplitudes and internal wave functions. Such studies should be performed in the future.

A detailed comparison has been carried out of our results with standard predictions of statistical reaction theory. The average cross section was compared with the Hauser-Feshbach formula for a large number of channels. In the inelastic case this description works quite well in the overlapping resonance regime for any interaction strength, while in the elastic case strong deviations have been found if the intrinsic motion is not fully chaotic.

The study of Ericson fluctuation theory shows that the assumption that the fluctuations of the resonance widths become negligible for a large number of channels is wrong in the overlapping regime. We found that the fluctuations of resonance widths increase with the coupling to the continuum, and we gave evidence that the relative fluctuation of the width (the ratio of the variance to the square of the average width) diverges at $\kappa=1$ for any number of channels. This should imply that for any number of channels the differences from the standard theory should increase as $\kappa$ increases.

In order to study the relationship between the variance of the cross section and its average value, it is necessary to take into account the dependence of the average cross section on the intrinsic interaction strength $\lambda$. Even when this is done, the standard prediction about the variance of the cross section was found to be a good approximation only for a very large number of channels. For $M$ between 10 and 20, where the Ericson prediction could be expected to be valid, consistent deviations have been demonstrated. In particular, the distribution of cross sections shows that the probability of a large value of the cross section, mainly for the elastic case (or in the presence of direct reactions), can be well below conventional predictions.

Finally, we have shown that, in agreement with previous studies, the correlation length differs from the average width for any number of channels. On the other hand, the Weisskopf relation (62) that connects the correlation length of the cross section to the transmission coefficient, works, for a large number of channels, at any value of the intrinsic interaction strength $\lambda$. In many situations we have seen that increase of $\lambda$ in fact suppresses the fluctuations in the continuum. This can be understood qualitatively as a manifestation of many-body chaos that makes all internal states uniformly mixed.

Our results can be applied to any many-fermion system coupled to the continuum of open decay channels. The natural applications first of all should cover neutron resonances in nuclei, where rich statistical material was accumulated but the transitional region from isolated to overlapped resonances was not studied in detail. The interesting applications of a similar approach to molecular electronics and electron tunneling spectroscopy can be found in the recent literature [42, 43]. Other open mesoscopic systems, for example, quantum dots and quantum wires, should be analyzed as well in the crossover region. One can expect very promising designated studies for checking the statistical properties of resonances in such controllable experiments as those in microwave cavities and in acoustical chaos. Open boson systems in atomic traps also can be an interesting object of future 
theoretical and experimental studies.

\section{ACKNOWLEDGMENT}

We acknowledge useful discussion with D. Savin, T. Kawano, V. Sokolov and T. Gorin. The work was supported by the NSF grants PHY-0244453 and PHY0555366. The work by G.P.B. was carried out under the auspices of the National Nuclear Security Administration of the U.S. Department of Energy at Los Alamos National Laboratory under Contract No. DE-AC5206NA25396. F.M.I. acknowledges partial support by the CONACYT (México) grant No 43730.
[1] W. Hauser and H. Feshbach, Phys. Rev. 87, 366 (1952).

[2] T. Ericson, Ann. Phys., 23, 390 (1963).

[3] D. Brink and R. Stephen, Phys. Lett. 5, 77 (1963).

[4] T. Ericson and T. Mayer-Kuckuk, Ann. Rev. Nucl. Sci. 16, 183 (1966).

[5] C. Mahaux and H.A. Weidenmüller, Shell Model Approach to Nuclear Reactions (North Holland, Amsterdam, 1969).

[6] D. Agassi, H.A. Weidenmüller, and G. Mantzouranis, Phys. Rep. 3, 145 (1975).

[7] J.J.M. Verbaarschot, H.A. Weidenmüller, and M.R. Zirnbauer, Phys. Rep. 129, 367 (1985).

[8] T.A. Brody, J. Flores, J.B. French, P.A. Mello, A. Pandey, and S.S.M. Wong, Rev. Mod. Phys. 53, 385 (1981).

[9] P.A. Moldauer, Phys. Rev. 157, 907 (1967).

[10] P.A. Moldauer, Phys. Rev. Lett. 23, 708 (1969).

[11] M. Simonius, Phys. Lett. B 52, 279 (1974).

[12] P. Kleinwächter and I. Rotter, Phys. Rev. C 32, 1742 (1985).

[13] V.V. Sokolov and V.G. Zelevinsky. Phys. Lett. B 202, 10 (1988).

[14] V.V. Sokolov and V.G. Zelevinsky, Nucl. Phys. A504, 562 (1989).

[15] V.V. Sokolov and V.G. Zelevinsky, Ann. Phys. (N.Y.) 216, 323 (1992).

[16] L. Durand, Phys. Rev. D 14, 3174 (1976).

[17] G. E. Brown and M. Bolsterli, Phys. Rev. Lett. 3, 472 (1959).

[18] V.V. Sokolov and V.G. Zelevinsky, Fizika (Zagreb) 22, 303 (1990).

[19] V.V. Sokolov, I. Rotter, D.V. Savin, and M. Müller, Phys. Rev. C 56, 1031, 1044 (1997).

[20] V. Zelevinsky and A. Volya, in Proceedings of the 11th Varenna Conference on Nuclear Reaction Mechanisms, ed. by E. Gadioli (Universita degli Studi di Milano, Supplemento N. 126, 2006) p. 73.

[21] R.H. Dicke, Phys. Rev. 93, 99 (1954).
[22] F.M. Izrailev, D. Sacher and V.V. Sokolov, Phys. Rev. E 49, 130 (1994).

[23] H.-J. Sommers, Y.V.Fyodorov, and M.Titov, J. Phys. A: Math. Gen. 32, L77 (1999).

[24] I. Rotter, Rep. Prog. Phys. 54, 635 (1991).

[25] A. Volya and V. Zelevinsky, Phys. Rev. Lett. 94 (2005) 052501 .

[26] A. Volya and V. Zelevinsky, in Nuclei and Mesoscopic Physics, ed. V. Zelevinsky, AIP Conference Proceedings 777, 2005, p. 229.

[27] F. Haake et al., Z. Phys. B 88, 359 (1992).

[28] S. Mizutori and V.G. Zelevinsky, Z. Phys. A346, 1 (1993)

[29] G.L. Celardo, F. Izrailev, V. Zelevinsky and G. Berman, to be published.

[30] A. Volya and V. Zelevinsky, Phys. Rev. C 74, 064314 (2006).

[31] V.V. Flambaum and F.M. Izrailev, Phys. Rev. E 56, 5144 (1997).

[32] T. Gorin and T.H. Seligman, Phys. Rev. E 65, 26214 (2002).

[33] V. Zelevinsky, B.A. Brown, N. Frazier, and M. Horoi, Phys. Rep. 276, 85 (1996).

[34] P.A. Moldauer, Phys. Rev. C 11, 426 (1975).

[35] T. Ericson, Phys. Rev. Lett. 5, 430 (1960).

[36] A. Muller and H.L. Harney, Phys. Rev. C 33, 1228 (1987).

[37] N.Lehmann, D.Saher, V.V. Sokolov and H.-J. Sommers, Nucl. Phys. A582, 223 (1995).

[38] E.D. Davis and D. Boose, Phys. Lett. B 211, 379 (1988).

[39] E.D. Davis and D. Boose, Z. Phys. A332, 427 (1989).

[40] F.M. Dittes, H.L. Harney and A. Muller, Phys. Rev. A, 45, 701 (1992).

[41] W.P. Abfalterer et al., Phys. Rev. C 62, 64312 (2000).

[42] I. Cacelli, A. Ferretti, M. Girlanda, and M. Macucci, Chem. Phys. 333, 26 (2007).

[43] K. Walczak, Chem. Phys. 333, 63 (2007). 